LA W RENCE LIVERM ORE NATIONAL LABORATORY
Lambda and Anti-Labmda production in the ALICE experiment at the LHC.

R. Carmona, R. Soltz

September 12, 2013 
This document was prepared as an account of work sponsored by an agency of the United States government. Neither the United States government nor Lawrence Livermore National Security, LLC, nor any of their employees makes any warranty, expressed or implied, or assumes any legal liability or responsibility for the accuracy, completeness, or usefulness of any information, apparatus, product, or process disclosed, or represents that its use would not infringe privately owned rights. Reference herein to any specific commercial product, process, or service by trade name, trademark, manufacturer, or otherwise does not necessarily constitute or imply its endorsement, recommendation, or favoring by the United States government or Lawrence Livermore National Security, LLC. The views and opinions of authors expressed herein do not necessarily state or reflect those of the United States government or Lawrence Livermore National Security, LLC, and shall not be used for advertising or product endorsement purposes.

This work performed under the auspices of the U.S. Department of Energy by Lawrence Livermore National Laboratory under Contract DE-AC52-07NA27344. 


\section{Strangeness Production in Jets with ALICE at the LHC}

Rodney Carmona, Mike Tyler II, Ron Soltz, Austin Harton, Edmundo Garcia

Chicago State University, Lawrence Livermore National Laboratory

\section{Cern (European Organization for Nuclear Research)}

At this laboratory Physicists and Engineers study fundamental particles using the world's largest and most complex instruments. Here particles are made to collide at close to the speed of light to see how they interact and help to discover the basic laws of nature. One type of instrument used at CERN is a particle accelerator that boosts beams of particles to high energies and make them collide with either stationary particles or each other. Other types of instruments are the detectors which observe and Soltz, Ron 9/11/13 4:54 PM Deleted: s Soltz, Ron 9/11/13 4:55 PM record the collisions. CERN was founded in 1954 and sits by the France-Swiss border near Geneva. It Deleted: Ano was one of Europe's first joint ventures and now has 20 member states.

The name CERN is an acronym for the French "Conseil Européen pour la Recherche Nucléaire", or European Council for Nuclear Research, a provisional body founded in 1952 with the mandate of establishing a world-class fundamental physics research organization in Europe. At that time, pure physics research concentrated on understanding the inside of the atom.

CERN's main area of research is particle physics the study of the fundamental constituents of matter and the forces acting between them. The laboratory operated by CERN is often referred to as the European Laboratory for Particle Physics.[1]

\section{LHC (Large Hadron Collider)}

The world's largest and most powerful particle accelerator first started up on September 10, 2008 and is the latest addition to CERN. The collider is a 27 kilometer ring of superconducting magnets with accelerating structures to boost energy. 


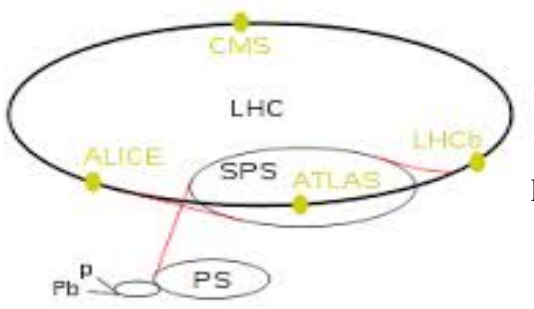

Fig. 5[22]

Inside the accelerator, two high-energy particle beams travel at close to the speed of light before they are made to collide. The beams travel in opposite directions in separate beam pipes - two tubes kept at vacuum. They are guided around the accelerator ring by a strong magnetic field maintained by superconducting electromagnets. The electromagnets are built from coils of special electric cable that operates in a superconducting state, efficiently conducting electricity without resistance or loss of energy. This requires cooling the magnets to $-271.3^{\circ} \mathrm{C}$. For this reason, much of the accelerator is connected to a distribution system of liquid helium, which cools the magnets, as well as supplying other services.

Thousands of magnets of different varieties and sizes are used to direct the beams around the accelerator. These include 1232 dipole magnets 15 meters in length which bend the beams, and 392 quadrupole magnets, each 5-7 meters long, which focus the beams. Just before the collision, another type of magnet is used to "squeeze" the particles closer together to increase the chances of collisions. The Cern Control Center controls and houses the accelerator, its services and technical infrastructure. From here, the beams inside the LHC are made to collide at four locations around the accelerator ring, corresponding to the positions of four particle detectors ATLAS, CMS, ALICE and LHCb. [2] 


\section{ATLAS}

A dual purpose that investigates a wide range of physics applications from searching for the Higgs Boson to particles that might be dark matter. Atlas has 6 different detecting systems that record path, momentum and energy that can uniquely identify each particle. ATLAS uses an advanced triggering system that filters unwanted data out before it is stored. ATLAS is a 7,000 ton detector and is the largest volume particle detector built. ATLAS is located near Meyrin, Switzerland $100 \mathrm{~m}$ beneath the ground. It has more than 3000 working scientists involved.[3]

\section{CMS (Compact Moun Solenoid)}

CMS is a general-purpose detector at the LHC. It is designed to investigate a wide range of physics, including the search for the Higgs boson, extra dimensions, and particles that could make up dark matter. Although it has the same scientific goals as the ATLAS experiment, it uses different technical solutions and a different magnet-system design.

The CMS detector is built around a huge solenoid magnet. This takes the form of a cylindrical coil of superconducting cable that generates a field of 4 Tesla, about 100,000 times the magnetic field of the Earth. The field is confined by a steel "yoke" that forms the bulk of the detector's 12,500-tonne weight. An unusual feature of the CMS detector is it was constructed in 15 sections at ground level before being lowered into an underground cavern near Cessy, France and reassembled.

The CMS experiment is one of the largest international scientific collaborations in history, involving 4300 particle physicists, engineers, technicians, students and support staff.[4]

\section{LHCb (Large Hadron Collider beauty)}

The LHCb studies the bottom quark or beauty quark to find the differences in matter and antimatter. LHCb experiment doesn't enclose the collision but instead uses a series of sub-detectors to detect 
mainly forward particles and those thrown forwards by the collision in one direction. To catch the rarer bottom quarks, $\mathrm{LHCb}$ has developed sophisticated movable tracking detectors close to the path of the beams circling in the LHC. The 5600-tonne $\mathrm{LHCb}$ detector is made up of a forward spectrometer and planar detectors. It sits below ground near village of Ferney-Voltaire, France. About 700 scientists work on the $\mathrm{LHCb}$ experiment. [5]

\section{ALICE (A Large Ion Collider Experiment)}

ALICE is a heavy-ion detector on the LHC ring. It is designed to study the physics of strongly interacting matter at extreme energy densities, where a phase of matter called quark-gluon plasma forms. No quark has ever been observed in isolation: the quarks, as well as the gluons, seem to be bound permanently together and confined inside composite particles, such as protons and neutrons.

This is known as confinement.[6]

For part of each year the LHC provides collisions between lead ions, recreating in the laboratory conditions similar to those just after the big bang. Under these extreme conditions, protons and neutrons "melt", freeing the quarks from their bonds with the gluons. This is quark-gluon plasma. The existence of such a phase and its properties are key issues in the theory of quantum chromodynamics (QCD), for understanding the phenomenon of confinement, and for a physics problem called chiral-

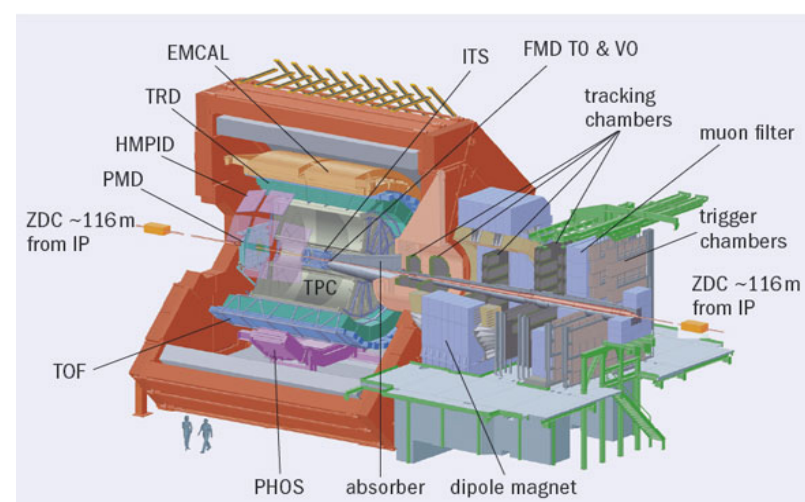

symmetry restoration. The ALICE

collaboration studies the quark-gluon

plasma as it expands and cools.

Fig. 6 [23]

The ALICE collaboration uses the 10,000-tonne ALICE detector to study 
quark-gluon plasma. The detector sits in a cavern $100 \mathrm{~m}$ below ground close to the village of St GenisPouilly in France, receiving beams from the LHC. The ALICE collaboration has more than 1000 scientists. [6]

Smaller LHC experiments

\section{TOTEM (Total elastic and diffractive cross-section measurement)}

TOTEM studies forward particles to focus on physics that is not accessible to the general-purpose experiments. It measures the size of the proton, and accurately monitors the luminosity of the LHC. Luminosity is the number particles per unit area per time, taking into account the target's penetrability to electromagnetic radiation.

To do this TOTEM must be able to detect particles produced very close to the LHC beams. It includes detectors housed in specially designed vacuum chambers called "Roman pots"' connected to the beam pipes in the LHC. Although the two experiments are scientifically independent, TOTEM complements the results from the CMS detector and from other LHC experiments.

The 20-tonne TOTEM detector is made up of gas-electron-multiplier detectors and cathode strip chambers, in addition to the 8 Roman pots. The experiment spans $440 \mathrm{~m}$ and is located below ground near the CMS detector in Cessy, France. [7]

\section{LHCf (Large Hadron Collider forward)}

The LHCf experiment uses particles thrown forward by collisions in the LHC as a source to simulate cosmic rays in laboratory conditions. Cosmic rays are naturally occurring charged particles from outer space that constantly bombard the Earth's atmosphere. They collide with nuclei in the upper atmosphere, triggering a cascade of particles that reaches ground level. Studying how collisions inside the LHC cause similar cascades of particles will help physicists to interpret and calibrate large-scale cosmic-ray experiments that can cover thousands of kilometers. 
LHCf is made up of two detectors which sit along the LHC beam-line, at 140 meters either side of the ATLAS collision point. The location allows the observation of particles at nearly zero degrees to the proton beam direction. Each of the two detectors weighs only 40 kilograms and involves 30 scientists.

[8]

\section{MOEDAL (Monopole and Exotics Detector at the LHC)}

MOEDAL was made to search directly for the magnetic monopole - a hypothetical particle with a magnetic charge.

The MOEDAL collaboration has built a detector to search for this particle. This monopole detector is an array of 400 modules, each consisting of a stack of 10 sheets of plastic nuclear-track detectors. This detector is deployed around the same intersection region as the $\mathrm{LHCb}$ detector.

MOEDAL is also looking for highly ionizing Stable Massive Particles (SMPs), predicted by theories beyond the Standard Model. [9]

\section{ALICE SubDetectors}

The ALICE experiment is comprised of the following sub-detectors:

\section{The Forward Multiplicity Detector (FMD)}

It is designed to measure the charged particles that are produced in the collisions and which are emitted at small angles relative to the beam line direction. [10]

\section{Zero Degree Calorimeters (ZDC)}

The ZDCs are calorimeters which detect the energy of the spectator nucleons in order to determine the overlap region of the two colliding nuclei. It is composed of four calorimeters, two to detect protons (ZP) and two to detect neutrons $(\mathrm{ZN})$. They are located 115 meters away from the interaction point on both sides, exactly along the beam line. That is why we call them Zero Degree Calorimeters (ZDC). [11] 


\section{Particle Trackers}

\section{Time-Projection Chamber (TPC)}

The TPC the main device in ALICE and is used for tracking of charged particles and particle identification. The ALICE TPC was designed to cope with the highest conceivable charged particle multiplicities predicted, at the time of the Technical Proposal, for central $\mathrm{Pb}-\mathrm{Pb}$ collisions at $\mathrm{LHC}$ energy. Its acceptance covers $2 \pi$ in azimuthal angle and a pseudo-rapidity interval $\mid$ eta $\mid<0.9$. Including secondaries, the above charged particle rapidity density could amount to 20000 tracks in one interaction in the TPC acceptance. [12]

\section{The Silicon Pixel Detector (SPD)}

The SPD is part of the Inner Tracking System (ITS) of the ALICE experiment. The purpose of the SPD is to provide ALICE with secondary vertexing capability for charm and beauty detection in such a high multiplicity environment (charged particle multiplicities of up to 8000 per unit of rapidity have been predicted for head-on $\mathrm{Pb}-\mathrm{Pb}$ collisions at the $\mathrm{LHC}$ ). The pseudorapidity coverage of the inner layer is $|\eta|<1.95$. The two SPD layers allow to achieve a track impact parameter resolution in the plane perpendicular to the beam axis better than $50 \mu \mathrm{m}$ for $\mathrm{pt}>1.3 \mathrm{GeV} / \mathrm{c}$. [13]

\section{The Transition Radiation Detector (TRD)}

The TRD is the main electron detector in ALICE. In conjunction with the TPC and the ITS, it provides the necessary electron identification capability to study the production of light and heavy vector mesons as well as the continuum in the di-electron channel and semi-leptonic decays of hadrons with open charm and open beauty. It can also study correlated DD and BB pairs and jets. [14]

\section{Particle Identifiers}

\section{The Di-moun Spectrometer}


Studies the complete spectrum of heavy quarkonia via decay in the mu and mu- channel. [15]

\section{The High Momentum Particle Identification (HMPID)}

The HMPID system will enhance the PID capability of ALICE beyond the momentum range allowed by the energy loss measurements and by the TOF. The HMPID detector has been designed to extend the useful range for the identification of $\mathrm{p}$ and $\mathrm{K}$ up to $3 \mathrm{GeV} / \mathrm{c}$ and of $\mathrm{p}$ up to $5 \mathrm{GeV} / \mathrm{c}$, on a track-bytrack basis. [16]

\section{Time of Flight Detector_(TOF)}

TOF detector detects charged particles with intermediate momentum. It uses time measurement, track length and the momentum to calculate the particles mass. A time resolution of $100 \mathrm{ps}$ will provide $3 \sigma$ $\pi / \mathrm{K}$ separation up to $2.2 \mathrm{GeV} / \mathrm{c}$ and $\mathrm{K} / \mathrm{p}$ separation up to $4 \mathrm{GeV} / \mathrm{c}$. The goal is to study -The QCD thermodynamics via the measurement of $\pi, \mathrm{K}$ and $\mathrm{p}$ transverse momentum distributions and particle ratios on an Event-by- Event basis. [17]

\section{Photon Catchers}

\section{Photon Spectrometer (PHOS)}

PHOS (PHOton Spectrometer) is a high resolution electromagnetic calorimeter consisting of 17920 detection channels based on leadtungstate crystals (PWO). [18]

The photons tell us about the temperature of the system. To measure them, special detectors are necessary: the crystals of the PHOS, which are as dense as lead and as transparent as glass, will measure them with precision in a limited region, while the PMD and in particular the EMCal will

measure them over a very wide area. The EMCal will also measure groups of collimated high energy particles (called "jets") which have a memory of the early phases of the event. 


\section{EMCal (Electromagnetic Calorimeter)}

Captures photons and electrons in a very wide region. Its main design is to study jet quenching and act as a trigger. It also has a high pseudorapidity. [19]

\section{PMD (Photon Multiplicity Detector)}

In the PMD, photons are detected via electromagnetic showers to which they convert in the 3 radiation lengths thick lead converter plates. The produced shower particles give rise to a large signal in $3 \mathrm{~mm}$ thick scintillator pads. These pads are read out by wavelength shifting optical fibers coupled to image intensifiers and CCD cameras. Hadrons, due to their large nuclear interaction length, will mainly traverse the converter without showering. Thus they will give only a small signal in the scintillator (as minimum ionizing particle), which gives a handle to discriminate them. [20]

Strangeness is the description of decay of particles in strong and electromagnetic reactions. The terms strange and strangeness were coined before the strange quark was even found. The way it fits in to heavy ion research is that it shows and predicts how particles will decay.[19] the lower strange amount cause the particles to decay at a slower rate using the weak interaction. The higher amount of strangeness causes the particles to decay at a faster rate using the strong force interaction. [fig.1] The reason this is so important for heavy ion research is that using this known data we can know exactly where a particle will decay at and be able to pinpoint its origin.

$$
S=-\left(n_{s}-n_{\bar{S}}\right) \text { Fig.1 [1] }
$$

In ALICE the Omega baryon particle has been measured using a proton-proton collision. The Omega baryon is a 3 strange quark particle. At ALICE there has been a higher number of Lambda, Xi, and Omega particles [fig. 2] using $\mathrm{Pb}-\mathrm{Pb}$ collisions. Using $\mathrm{Pb}-\mathrm{Pb}$ collisions at ALICE more particles 
can be observed even at lower energies.[20] This greater yield of data allows more theories to be tested at a faster rate and with more accuracy. This shows how ALICE is a frontier in strangeness research.

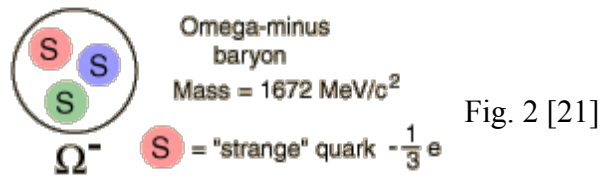

We will be measuring Lambda and K short Invariant Mass plots and examining the data. After examining I will decide where to make the proper cuts to the data to come up with clear pictures of the

Lambda and K short plots, I will also be measuring new distribution charts and histograms that I will create with the altered invariant mass plots. After measuring the new distribution histograms they will also be cut and altered to have the need data come through the background. After all plots have been measured and analyzed I will find the centrality and the transverse momentum of the particles.

What I expect to get from this research is a basic understanding on how particle physics is studied and analyzed in an international laboratory such as CERN. I also hope to achieve a greater grasp on how to use $\mathrm{C}++$ as a tool to study physics and model mathematically what is happening inside these major detectors at LHC. One other aspect I hope to gain from this research is how to cooperate with other workers who have different working and learning styles. The main point I will take away from this research is the ability to continue after I'm done and be a proficient contributor to the ALICE experiment and to one day discover something new.

\section{Methodology}

The methodology of getting the correct data out of the AliAOD files is as follows. First, have a newly installed aliroot program on a Linux or Mac machine. Next, download the files that need to be analyzed from the grid and link them to a local test file. Then, create a .C file that can read the AOD files and create .cxx and .h files in the same directory. Add new histograms to .cxx file to analyze all AOD data. Furthermore, look up and use the root libraries to analyze the data with vertex z, DCA,TPC, 
etc.. Then, Use if statements to filter out noise from each histogram and separate each function by Lambda and K0Short. Next, separate the background noise and signal using Gaussian and Polynomial functions. Finally, re-insert signal on top of background and find the integral of the space between signal and background to show how many particles there are.

\section{Results}

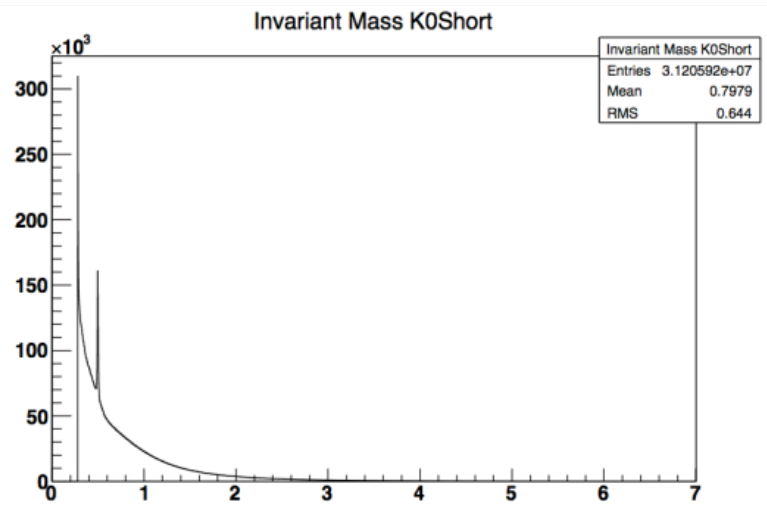

Fig. (a) Original Signal

Using the Vertex 0 finder, invariant mass for the Lambda, anti-Lambda, and K0Short_(Fig(a)) are easily obtainable with a high level of clarity. These histograms are the beginning of the analysis to finding how many particles are in the data. 


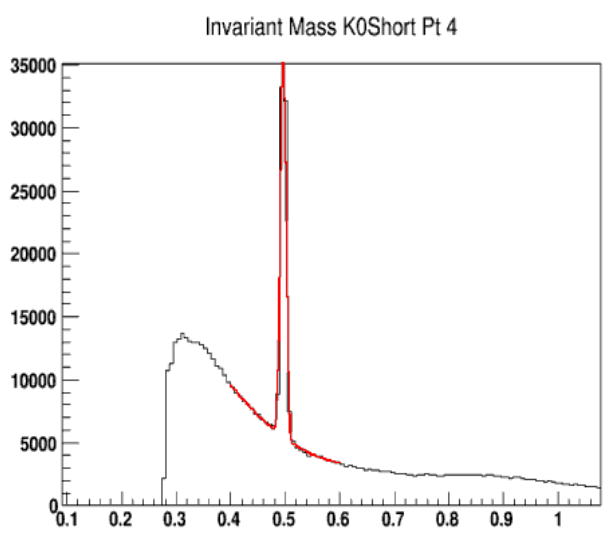

Fig(b) Signal with 3rd Order Polynomial

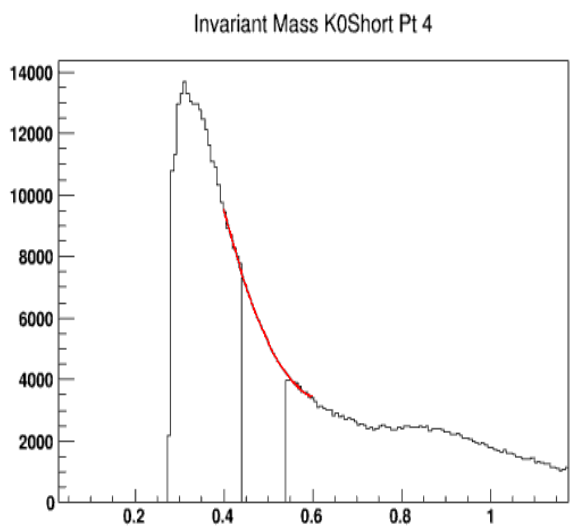

Fig(c) Background with Gaussian function

Isolating the signal from the background in each momentum bin and applying a Polynomial function

(Fig(b)) over the data is the first step. Then, subtracting the signal from the background and applying a Gaussian function over it (Fig(c)).

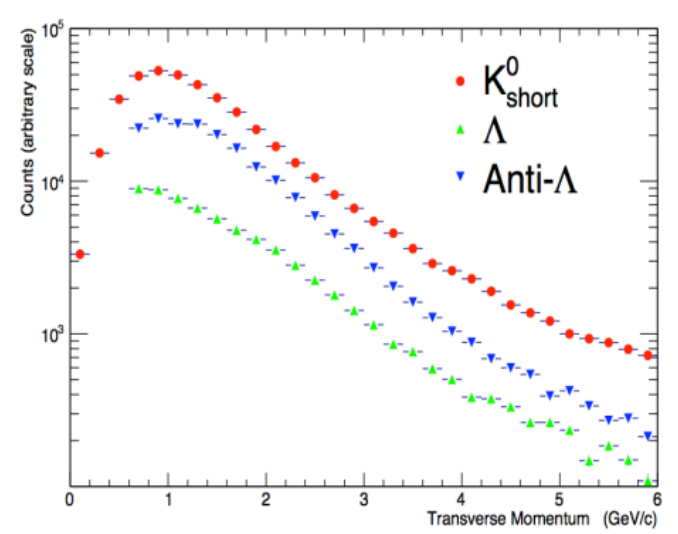

Fig(d) Number of particles per Momentum Bin

After all momentum bins are analyzed for each of the Lambdas, Anti-Lambdas, and K0Shorts, 
particles per momentum bin. All results are then plotted on a new histogram (Fig(d)).

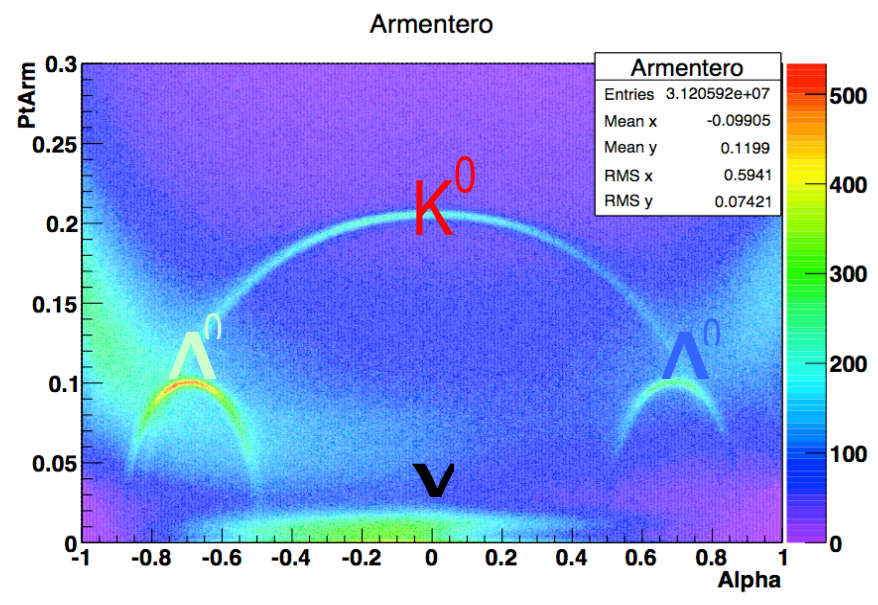

Fig(e) Armentero Plot

Armentero distribution is a quality check of the V0. It allows us to show the different identified particle bands.

\section{Conclusion / Further Study}

In conclusion, using prescribed methods have yield clear results and numbers. The results obtained indicate that the signal cuts performed are reducing background noise and showing a clearer signal. For further study efficiencies need to be constructed and analyzed in a simulation with regards to the same cuts that have been applied to the original data. This will further improve the signal and background ratio as well as verify the amount of particles that are present inside the AOD files.

Another area of further study after efficiencies are completed is the study of the production of jets. 


\section{References:}

[1] http://home.web.cern.ch/about

[2] http://home.web.cern.ch/about/accelerators/large-hadron-collider

[3] http://home.web.cern.ch/about/experiments/atlas

[4] http://home.web.cern.ch/about/experiments/cms

[5] http://home.web.cern.ch/about/experiments/lhcb

[6] http://home.web.cern.ch/about/experiments/alice

[7] http://home.web.cern.ch/about/experiments/totem

[8] http://home.web.cern.ch/about/experiments/lhcf

[9] http://home.web.cern.ch/about/experiments/moedal

[10] http://aliceinfo.cern.ch/Public/en/Chapter2/Chap2_FMD.html

[11] http://aliceinfo.cern.ch/Public/en/Chapter2/Chap2_ZDC-en.html

[12] http://aliceinfo.cern.ch/TPC/

[13] http://aliceinfo.cern.ch/Public/en/Chapter2/Chap2_SPD.html

[14] http://aliceinfo.cern.ch/Public/en/Chapter2/Chap2_TRD.html

[15] http://aliceinfo.cern.ch/Public/en/Chapter2/Chap2_dim_spec.html

[16] http://aliceinfo.cern.ch/Public/en/Chapter2/Chap2_HMPID-en.html 
[17] http://aliceinfo.cern.ch/Public/en/Chapter2/Chap2_TOF.html

[18] http://aliceinfo.cern.ch/Public/en/Chapter2/Chap2_phos.html

[19] http://en.wikipedia.org/wiki/Strangeness

[20] http://alicematters.web.cern.ch/?q=abelevbetty_strangeness

[21] http://hyperphysics.phy-astr.gsu.edu/hbase/particles/omega.html

[22] http://en.wikipedia.org/wiki/Compact_Muon_Solenoid

[23] http://cerncourier.com/cws/article/cern/35879

[19] https://trac.cc.jyu.fi/projects/alice/wiki/EMCAL

[20] http://www.uni-muenster.de/Physik.KP/santo/thesis/phd/schlagheck/node21.html 